\title{
Short Cavity DFB Fiber Laser Based Vector Hydrophone for Low Frequency Signal Detection
}

\author{
Xiaolei ZHANG, Faxiang ZHANG, Shaodong JIANG, Li MIN, Ming LI, \\ Gangding PENG, Jiasheng NI, and Chang WANG*
}

\author{
Shandong Key Laboratory of Optical Fiber Sensing \& Techonology, Laser Institute of Shandong Academy of Science, \\ China, Jinan, 250014, China \\ *Corresponding author: Chang WANG_E-mail: bluestone469@hotmail.com
}

\begin{abstract}
A short cavity distributed feedback (DFB) fiber laser is used for low frequency acoustic signal detection. Three DFB fiber lasers with different central wavelengths are chained together to make three-element vector hydrophone with proper sensitivity enhancement design, which has extensive and significant applications to underwater acoustic monitoring for the national defense, oil, gas exploration, and so on. By wavelength-phase demodulation, the lasing wavelength changes under different frequency signals can be interpreted, and the sensitivity is tested about $33 \mathrm{~dB} \mathrm{re} \mathrm{pm} / \mathrm{g}$. The frequency response range is rather flat from $5 \mathrm{~Hz}$ to $300 \mathrm{~Hz}$.
\end{abstract}

Keywords: Short cavity distributed feedback fiber laser; vector hydrophone; frequency response; phase noise; sensitivity

Citation: Xiaolei ZHANG, Faxiang ZHANG, Shaodong JIANG, Li MIN, Ming LI, Gangding PENG, et al., "Short Cavity DFB Fiber Laser Based Vector Hydrophone for Low Frequency Signal Detection," Photonic Sensors, 2017, 7(4): 325-328.

\section{Introduction}

The underwater seismic wave stimulated by ship noise or marine meteorology environment has significant application prospects to underwater target detection, mine fuze design, ocean dynamic monitoring, etc. The current anti-submarine hydrophone has been challenged by radiation noise decrease of marine [1, 2]. In oceanography, the low frequency and ultra-low frequency acoustic signal has little attenuation and better space time coherence in propagation. As a result, deep research on low frequency towed line array and seabed fixed line array has become a popular topic [3].

Traditional piezoelectric ceramic hydrophone requires underwater electrical equipment and cable for multiplexing, data transferring, and power supplying, which is expensive and heavy, and has underwater sealing problems. The hydrophone array, used for low frequency signal detection, usually has huge volume, which causes various inconveniences in engineering application and cost increase.

All-fiber hydrophone array has no electric equipment underwater, large multiplexing capability, and anti-electromagnetic interference. Fiber vector hydrophone measures the three orthogonal components synchronously and concurrently of the acoustic pressure and particle velocity at a certain point of the sound field. Compared with the pressure hydrophone, the vector hydrophone obtains more information and provides more possibilities for subsequent data processing, which is crucial to low 
frequency signal acquisition, long-distance target detection, and location [4-6].

Many researches on fiber-optic flexural disk accelerometers have been reported in recent decades [7, 8], while fiber laser based accelerometers have become more and more attractive on account of high resolution and small volume $[9,10]$. We use a short cavity distributed feedback (DFB) fiber laser as the sensing element, to construct a vector hydrophone with a diameter of $10 \mathrm{~cm}$. Theoretical and experimental analyses have been carried out to illustrate a flat response from $5 \mathrm{~Hz}$ to $300 \mathrm{~Hz}$.

\section{Design and theory}

Three identity DFB fiber laser accelerators are assembled together to construct three-element co-oscillating type vector hydrophone as shown in Fig. 1. To control its attitude in water, the other three non-active accelerators are amounted therein to maintain balance. The diameter of the vector hydrophone is $10 \mathrm{~cm}$, and most importantly the total mass is controlled with the average density of about 1.8 times of water density, to guarantee the globe oscillating in the sound field with the same amplitude and phase [1].

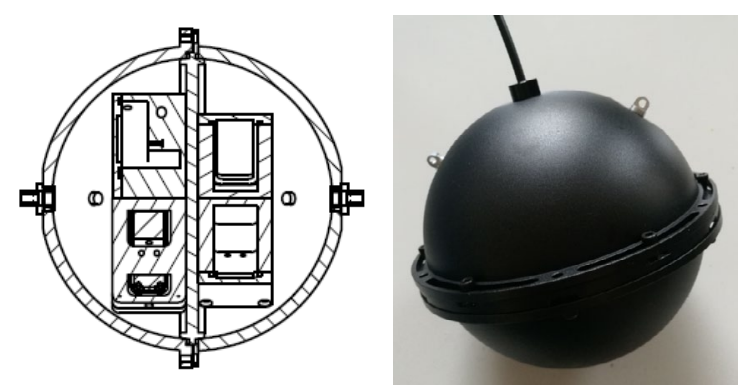

Fig. 1 DFB fiber laser based vector hydrophone.

Each element in the vector hydrophone is designed based on the cantilever beam structure. The stainless steel mass is fixed to the supporter by a spring strip as the inertial sensor. Then the DFB fiber laser is fixed between the supporter and the strutbeam of the mass. The outer vibration signal turns the mass, then the strutbeam pulls the fiber in order to change the axis strain on it, consequently the lasing wavelength of the DFB fiber laser will change.

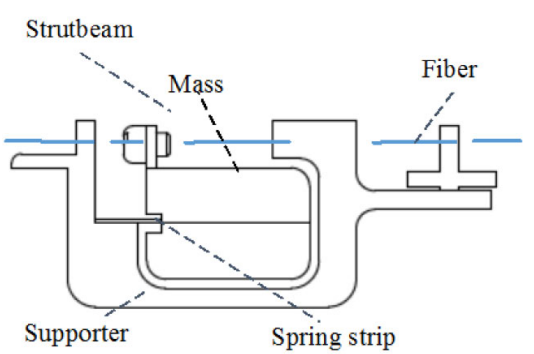

Fig. 2 Single element structure of vector hydrophone.

The acceleration sensitivity of single element of the DFB fiber laser vector hydrophone equals the ratio of wavelength change to acceleration, which is proportional to the quality of the mass $m$ and inversely proportional to the elasticity $K$ of the spring strip, as described in (1), where $h$ is the transfer function of the mass and the fiber. The resonant frequency is contrary to the sensitivity derived from (2) [11]. According to theory, the sensitivity is restricted to the response bandwidth. In our work, the resonant frequency is designed to be around $250 \mathrm{~Hz}$.

$$
\begin{gathered}
S=\frac{\Delta \lambda}{a} \propto h \frac{m}{K} \\
f_{0}=\sqrt{K / m} .
\end{gathered}
$$

The lasing wavelength change of DFB fiber laser $\Delta \lambda$ is transferred into phase difference change $\Delta \varphi$ :

$$
\Delta \phi=\frac{2 \pi n d \Delta \lambda}{\lambda_{B}^{2}}
$$

where $n$ is the refractive index of fiber, $d$ is the optical path difference, and $\Delta \varphi$ can be obtained by the phase demodulation technology. The DFB fiber laser we fabricated has a narrow linewidth of $10 \mathrm{kHz}$, which realizes the high resolution wavelength detection.

\section{Experiment and analysis}

\subsection{Calibration system}

The system we apply for calibration is drawn in Fig. 3. A commercial pump source is used. A non-balanced Michelson interferometer is used to transfer wavelength change into phase difference change and detected by a photoelectric detector. 
Then a commercial OPD4000 (optical phase demodulation) is applied together with the PGC (phase generation carrier) algorithm to restore the wavelength change [12].

A vibrostand JZ-5 is used where both the DFB fiber laser hydrophone and a standard piezoelectric accelerator are mounted.

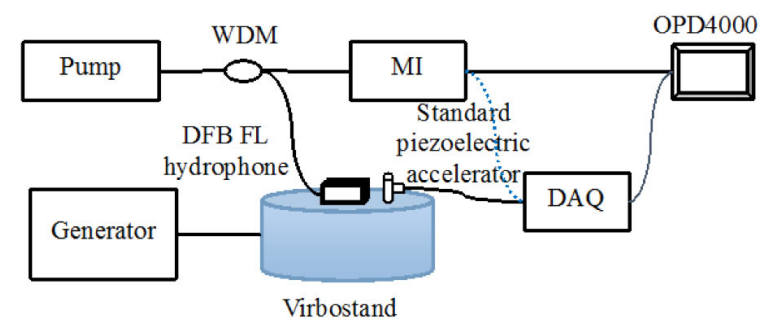

Fig. 3 Experimental setup for calibration of the DBF fiber laser hydrophone.

\subsection{Experimental results}

Theoretically, the wavelength resolution partly depends on the linewidth of DFB fiber laser, and the minimized measurable signal depends on the phase noise of the DFB fiber laser. It is indicated besides the sensor packaging design the vector hydrophone performance can be promoted by tailoring the DFB fiber laser design or fabrication parameter, such as lasing cavity length and ultraviolet (UV) exposure. Three DFB fiber lasers with different lasing wavelengths are chained together to realize a vector hydrophone, which are $1535.03 \mathrm{~nm}, 1539.77 \mathrm{~nm}$, and $1549.37 \mathrm{~nm}$ separately. The cavity length of the DBF fiber laser we fabricated is only $27 \mathrm{~mm}$ restricted to the diameter of the hydrophone cell, the phase noise is about $-120 \mathrm{~dB}$, the RIN (relative intensity noise) is $-90 \mathrm{~dB}$, the linewidth is about $10 \mathrm{kHz}$, and the output power balance is less than $3 \mathrm{~dB}$.

The DFB fiber laser vector hydrophone we have designed is calibrated by comparison with the data of the standard piezoelectric accelerator using the system described above. The acceleration sensitivity is measured to be about $40 \mathrm{~dB}$ re $\mathrm{pm} / \mathrm{g}$ from $5 \mathrm{~Hz}$ to $100 \mathrm{~Hz}$ without damping as shown in Fig. 4. To suppress the resonance peal as well as expand the response bandwidth, an appropriate damper should be added into the system. This procedure is accomplished in experiment, because the numerical simulation has major error compared with the practical situation.

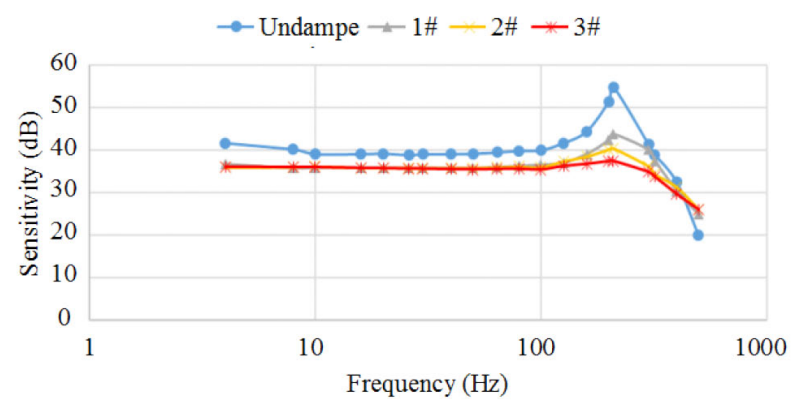

Fig. 4 Frequency response under different damping factors of the DFB fiber laser hydrophone.

Cases 1\# and 2\# apply silicon oil with the viscosity of $100 \mathrm{cst}, 350 \mathrm{cst}$ separately, while Case $3 \#$ applies silicon oil mixed of $350 \mathrm{cst}$ and $800 \mathrm{cst}$. The experimental results in Fig. 4 show that the sensitivity drops about $3 \mathrm{~dB}$ after adding silicon oil, and the larger viscosity brings out more flat frequency response with a resonance peak being suppressed more efficiently. However, the sensitivity keeps unchanged under $100 \mathrm{~Hz}$ because the silicon oil we used have the viscosity of little difference. In the optimized situation of Case 3\#, the flat bandwidth of response is expanded to $300 \mathrm{~Hz}$ successfully.

The frequency responses of three elements are tested and have good consistency as shown in Fig. 5.

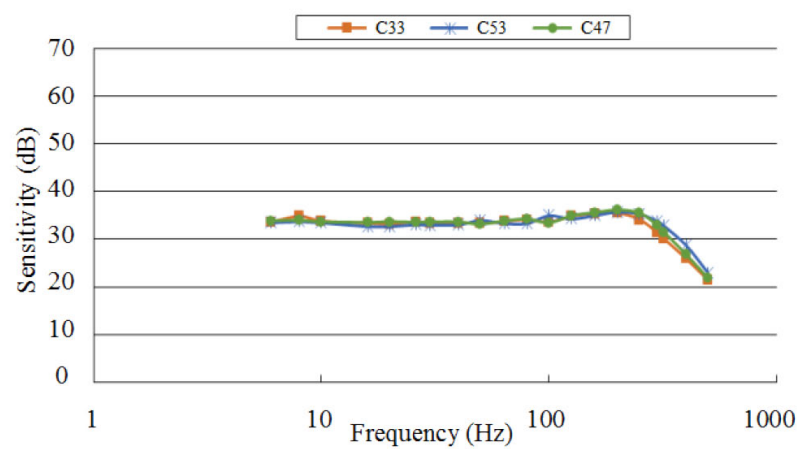

Fig. 5 Frequency response of the three-element DFB fiber laser hydrophone.

The DFB fiber laser hydrophone we design is capable of flat response with the sensitivity of about $33 \mathrm{~dB}$ re $\mathrm{pm} / \mathrm{g}$ from $5 \mathrm{~Hz}$ to $300 \mathrm{~Hz}$, with the 
fluctuation within $\pm 1 \mathrm{~dB}$.

\section{Conclusions}

A three-element vector hydrophone has been manufactured by short cavity DFB fiber lasers with the narrow linewidth with the proper sensitivity enhancement design. The lasing wavelength change under different frequency signals can be interpreted by unbalanced Michelson interferometer, and the sensitivity is tested to be about $33 \mathrm{~dB}$ re $\mathrm{pm} / \mathrm{g}$. The frequency response range is rather flat from $5 \mathrm{~Hz}$ to $300 \mathrm{~Hz}$. The low frequency signal detection capability allows extensive and significant applications in underwater acoustic monitoring in the national defense, oil, gas exploration and so on.

\section{Acknowledgment}

This work is supported by Shandong Key Research and Development Projects (No. 2015GSF115006), National Nature Science Foundation (No. 61605102), and Young Science Foundation of Shandong Academy of Sciences (No. 2016QN002).

Open Access This article is distributed under the terms of the Creative Commons Attribution 4.0 International License (http://creativecommons.org/ licenses/by/4.0/), which permits unrestricted use, distribution, and reproduction in any medium, provided you give appropriate credit to the original author(s) and the source, provide a link to the Creative Commons license, and indicate if changes were made.

\section{References}

[1] Z. G. Chen, "Marine radiation noise measurement technology based on vector hydrophone," Master dissertation, Harbin Engineering University, Harbin,
China, 2008.

[2] Y. Ma, "Development tendency of the world ocean and the influence on china," International Review, 2012, 4: 29-34.

[3] R. H. Min and J. X. Xiao, "The state and development of submarine integrated sonar systems around the world," Ship Science and Technology, 2013, 35(2): 134-141.

[4] A. Nehorai and E. Paldi, "Acoustic vector-sensor array processing," IEEE Transactions on Signal Processing, 1994, 42(9): 2481-2491.

[5] Y. Lv, "Research on key technologies of ultra-low frequency vector hydrophone system for subsurface buoy detection," Ph.D. dissertation, Harbin Engineering University, Harbin, China, 2010.

[6] L. F. Fu, P. X. Dou, Y. Chen, S. Q. Ma, and Z. Meng, "The directionality of shallow ambient noise using single optical fiber vector hydrophone," Technical Acoustics, 2013, 32(6): 105-106.

[7] G. A. Cranch and P. J. Nash, "High-responsivity fiber-optic flexural disk accelerometers," Journal of Lightwave Technology, 2000, 18(9): 1233-1243.

[8] G. Y. Chen, X. L. Zhang, G. Brambilla, and T. P. Newson, "Theoretical and experimental demonstrations of a microfiber-based flexural disc accelerometer," Optics Letters, 2011, 36(18): 3669-3671.

[9] Q. Jiang, Q. M. Sui, Y. C. Xu, H. G. Du, and D. B. Hu, "Design and experiments on distributed fiber laser hydrophone," Acta Photonica Sinica, 2009, 38(11): 2795-2799.

[10] L. N. Ma, Y. M. Hu, H. Luo, X. L. Zhang, and Z. Meng, "Acoustic pressure sensitivity of $\mathrm{Yb} /$ ErCo-doped distributed Bragg reflection fiber laser hydrophone," Chinese Journal of Lasers, 2009, 36(6): 1473-1478.

[11] F. X. Zhang, J. S. Lv, S. D. Jiang, B. X. Hu, X. L. Zhang, Z. Sun, et al., "High sensitive fiber Bragg grating micro-vibration sensor with shock resistance," Infrared and Laser Engineering, 2016, 45(8): 0822002-10822002-6.

[12] Q. Lin, L. H. Chen, S. Li, and X. Wu, "A high-resolution fiber optic accelerometer based on intracavity phase-generated carrier (PGC) modulation," Measurement Science and Technology, 2011, 22(22): 1-6. 